\title{
Ultrasound and microbubble-assisted gene delivery in Achilles tendons: Long lasting gene expression and restoration of fibromodulin $\mathrm{KO}$ phenotype
}

\author{
Anthony Delalande a,b, Ayache Bouakaz b, Gilles Renault c,d, Flore Tabareau e, Spiros Kotopoulis ${ }^{\mathrm{f}}$, \\ Patrick Midoux ${ }^{\text {a }}$, Brigitte Arbeille ${ }^{\mathrm{g}}$, Rustem Uzbekov ${ }^{\mathrm{g}}$, h, Shukti Chakravarti ${ }^{\mathrm{i}}$, \\ Michiel Postema ${ }^{\mathrm{j}}$, Chantal Pichon ${ }^{\mathrm{a}, *}$
}

a Centre de Biophysique Moléculaire UPR 4301 CNRS, rue Charles Sadron 45071 Orléans Cedex 2, France

b INSERM U930 CNRS ERL 3106, Université François-Rabelais Tours, France

' Institut Cochin, Université Paris Descartes, CNRS (UMR 8104), Paris, France

d Inserm, U1016, Paris, France

e CHR, Service d'anatomie et cytologie pathologiques, avenue de l'Hôpital, Orléans, France

${ }^{\mathrm{f}}$ Department of Engineering, The University of Hull, Cottingham Road, Kingston upon Hull, HU6 7RX, United Kingdom

${ }^{g}$ Département des Microscopies, Université François Rabelais, Tours, France

${ }^{\mathrm{h}}$ Faculty of Bioengineering and Bioinformatics, 119991, Moscow State University, Moscow, Russia

i Department of Medicine, Johns Hopkins School of Medicine, Baltimore, MD21205, USA

j University of Bergen, Institute of Physics and Technology, Allégaten 55, 5007 Bergen, Norway

\section{A R T I C L E I N F O}

\section{Article history:}

Received 6 June 2011

Accepted 14 August 2011

Available online 23 August 2011

\section{Keywords:}

Gene delivery

Sonoporation

Tendon

\begin{abstract}
A B S T R A C T
The aim of this study is to deliver genes in Achilles tendons using ultrasound and microbubbles. The rationale is to combine ultrasound-assisted delivery and the stimulation of protein expression induced by US. We found that mice tendons injected with $10 \mu \mathrm{g}$ of plasmid encoding luciferase gene in the presence of $5 \times 10^{5}$ BR14 microbubbles, exposed to US at $1 \mathrm{MHz}, 200 \mathrm{kPa}, 40 \%$ duty cycle for $10 \mathrm{~min}$ were efficiently transfected without toxicity. The rate of luciferase expression was 100-fold higher than that obtained when plasmid alone was injected. Remarkably, the luciferase transgene was stably expressed for up to 108 days. DNA extracted from these sonoporated tendons was efficient in transforming competent $E$. coli bacteria, indicating that persistent intact pDNA was responsible for this long lasting gene expression. We used this approach to restore expression of the fibromodulin gene in fibromodulin $\mathrm{KO}$ mice. A significant fibromodulin expression was detected by quantitative PCR one week post-injection. Interestingly, ultrastructural analysis of these tendons revealed that collagen fibrils diameter distribution and circularity were similar to that of wild type mice. Our results suggest that this gene delivery method is promising for clinical applications aimed at modulating healing or restoring a degenerative tendon while offering great promise for gene therapy due its safety compared to viral methods.
\end{abstract}

(c) 2011 Elsevier B.V. All rights reserved.

\section{Introduction}

Gene delivery by non-viral methods is a major challenge. Nonviral gene delivery methods are considered safer than viral gene delivery methods. The main limiting factor for non-viral gene therapy is the lack of efficiency due to suitable vectors.

Sonoporation is the transient permeabilization and resealing of a cell membrane with the help of ultrasound (US) and/or an ultrasound contrast agent, allowing for the trans-membrane delivery and cellular uptake of macromolecules between $10 \mathrm{kDa}$ and $3 \mathrm{MDa}$ [1-4]. Upon US exposure, microbubbles (MB) expand [5], move [6] and even fragment [4] which can modify the cell membrane permeability. This technique

\footnotetext{
* Corresponding author. Tel.: + 33 238255595; fax: + 33238631517. E-mail address: chantal.pichon@cnrs-orleans.fr (C. Pichon).
}

allows a site-specific local delivery of drugs or genes. Although the mechanism of membrane permeabilization has not been clearly understood, the main hypothesis of sonoporation assumes the formation of transient pores in the plasma membrane as a consequence of interaction of oscillating microbubbles with the cell membrane [3-5]. Transient pores formed at the plasma membrane are supposed to be responsible for the intracellular delivery of molecules but also the outward transport of intracellular molecules [7]. Recently, a study has shown that the endocytosis process could also be involved in the sonoporation mechanism for large molecules [8].

In the field of gene transfer, several studies have reported an improvement of gene delivery in the presence of US and MB [9]. Numerous studies on sonoporation and its exploitation for gene transfer have been reported $[10,11]$. This method has been applied to several organs including muscle and bone but its application to tendons has not been reported yet [3,12-19]. 
This study explores the use of sonoporation on Achilles tendons in vivo. We present results on the efficiency and ability to restore the expression of a "knocked out" gene. Tendons are essential as they connect muscles to bones allowing the movement around the joints. As musculoskeletal diseases, tendon defects constitute a major part of musculoskeletal disease and are one of the major public health issues in different countries.

Gene transfer approaches have been tested in tendons. Publications related to gene transfer applied to tendon pathologies show the potentiality of gene therapy $[20,21]$.

These include the use of adenoviral vectors [8,21], Hemagglutinin Japan Virus based-liposomes [22,23] and electroporation [24] as delivery modalities.

Adenoviral vector-mediated gene transfer has shown expression of the ß-galactosidase gene for 75 days [22]. Jayankura et al. investigated whether electrotransfer could improve gene transfer into mice or rat patellar tendons injected with pDNA alone [23]. They found that under specific conditions ( $200 \mathrm{~V}$ for $10 \mathrm{~ms}$ or $1200 \mathrm{~V}$ for $100 \mu \mathrm{s}$ ) gene expression could be improved by $50 \%$ when compared to pDNA injection alone. However, there was a gradual decrease and subsequent loss in gene expression after 42 days as was the case for pDNA injection alone.

In addition to sonoporation, therapeutic ultrasound has been claimed to promote protein synthesis in various tissues including in tendons [24-26]. In these studies, low intensity pulsed ultrasound (LIPUS) was used periodically to stimulate tissue remodeling or regeneration. LIPUS generally entails a 20 min treatment per day of $1 \mathrm{MHz}$ US at pulsed repetition frequency of $1 \mathrm{kHz}$ with an average intensity of $30 \mathrm{~mW} / \mathrm{cm}^{2}$.

Damaged muscle fibers have also been observed following in vivo sonoporation of mouse skeletal muscles [27]. In this study, the extent of the damaged muscle area was also significantly increased in the presence of OptisonTM microbubbles with or without P85, a block copolymer used as an adjuvant.

The expression of transgene in muscles can be enhanced by $1 \mathrm{MHz}$ US exposure alone at $2.5 \mathrm{~W} / \mathrm{cm}^{2}[3]$ and $3 \mathrm{~W} / \mathrm{cm}^{2}$ [28]. The discrepancy between these different studies could be due to the differences in the acoustic set up and in the applied acoustic conditions used and/or the age of animals used for the experiments. Lu et al. [28] have found that the effect of US alone on gene transfer in muscles was age-dependent.

Mechanical stress is known to induce some metabolic activities including gene expression and the mechanism through which US facilitates tissue repair is likely to be related to the mechanical effects of US waves $[29,30]$. These effects have been shown to produce a change in the membrane permeability and to stimulate the transport of second messenger substances, such as calcium across the cell membrane [29,31]. It has been observed that exposure to ultrasonic energy stimulates the proliferation of fibroblasts [32,33] and increases their metabolic activity [32-35]. A previous study has also reported that cell survival can be even increased at low duty cycle after sonoporation [36].

Our previous study showed the potential use of ultrasound and microbubbles for targeted gene delivery [28]. We used low intensity pulsed ultrasound to enhance gene delivery at the area of interest.

Here, we present the optimal acoustic conditions that allow localized and prolonged gene expression in Achilles tendons without inflammation or necrosis. Furthermore, we show the use of this method to restore expression of the Fmod gene in a fibromodulin KO mouse model highlighting the potentiality of this emerging gene delivery method for clinical gene transfer applications.

\section{Materials and methods}

\subsection{Reagents}

All reagents used in this study were purchased from SIGMAAldrich Co. (St Louis, MO, USA) unless otherwise stated.

\subsection{Plasmids}

A $7.5 \mathrm{~kb}$ homemade reporter plasmid that encoded the firefly luciferase gene (pLuc) under the control of the strong cytomegalovirus promoter was used. Five consecutive NFKB motifs that recognized the $\mathrm{NF} \kappa \mathrm{B}$ transcription factor were inserted upstream of the promoter [37]. A second plasmid which encodes the human fibromodulin was used $\mathrm{pCEP}_{4}$-FBM (pFmod) [38].

Supercoiled plasmid was isolated from Escherichia coli DH5 $\alpha$ bacteria cultures using the standard alkaline lysis method. Purification was carried out using a QIAGEN Mega Kit (QIAGEN, Courtabœuf, France).

\subsection{Microbubbles and ultrasound set-up}

BR14 (Bracco research, Geneva, Switzerland) and Micromarker® (VisualSonics, La Frette S/seine, France) microbubbles were used. Both contrast agents contain a perfluorobutane $\left(\mathrm{C}_{4} \mathrm{~F}_{10}\right)$ gas encapsulated by a phospholipid shell and have a median diameter of 2.3 to $2.9 \mu \mathrm{m}$. Both contrast agents were re-suspended according to manufacturer's protocol. The BR14 suspension contained approximately $2.10^{8} \mathrm{MB}_{\mathrm{ml}} \mathrm{ml}^{-1}$ whereas the Micromarker ${ }^{\circledR}$ contained $2.10^{9} \mathrm{MB}^{-\mathrm{ml}^{-1}}$. The experimental set up for tendon insonation was done as described as in Delalande et al. [39]. US was generated from a 0.5" diameter, IBMF-014 transducer with a center frequency of $1 \mathrm{MHz}$ (Sofranel, Sartrouville, France). A signal consisting of 40 cycles with a center frequency of $1.0 \mathrm{MHz}$ and a pulse repetition frequency of $10 \mathrm{kHz}$, i.e., a duty cycle of $40 \%$, was generated by a 33220A arbitrary function generator (Agilent technologies, Les Ulis, France) and amplified by a RF power amplifier (ADECE, Artannes, France) was used as the input for the transducer. The transducer was calibrated in a Perspex container using an HGL-200 PVDF bullet type hydrophone (Onda, Sunnyvale, CA). Peak negative acoustic pressures of $200 \mathrm{kPa}$ and $400 \mathrm{kPa}$ corresponding to an $\mathrm{MI}$ of 0.2 and 0.4 respectively were used.

\subsection{Animal studies}

The animal studies were carried out according to the guidelines of the French Ministry of Agriculture for experiments with laboratory animals (law 87848, C. Pichon accreditation). Mice were housed in the animal facility of our institution and cared for in conformity with the guidelines of Ethics Committee. Experiments were done on six to eight week old wild type (WT) CD1 mice and fibromodulin knock-out mice (Fmod KO).

Mice were anesthetized using $2-3.5 \%$ isoflurane inhalation. The posterior legs of the mice were shaved and washed with Vetedine $(\subset$ (Centravet, Alfort, France).

A $10 \mu$ solution containing $10 \mu \mathrm{g}$ of pDNA and $5 \times 10^{5} \mathrm{MB}$ was injected into the Achilles tendon area using a 29G Myjector syringe (Terumo France SA, Guyancourt, France). US stimulation was performed immediately after injection. Control experiments were performed using $10 \mu \mathrm{l}$ of pDNA alone on a separate mouse or on the other posterior leg. The transducer was positioned as so its natural focus coincided with the mouse skin.

The presence of MB close to the tendon was assessed using highresolution ultrasound imaging. Images of the tendon and surrounding muscle tissue were captured using a RMV-708 probe of a VEVO770 (Visualsonics) ultrasound biomicroscope.

US images with a $40 \mu \mathrm{m}$ axial resolution were captured before and after injection of $10 \mu$ solution containing $5 \times 10^{5}$ BR14 microbubbles.

MB were displayed as highly echogenic areas. Additional images of the tendon and leg after insonation were acquired to assess if any microbubbles remained.

To ensure that the remaining hyper echogenic structures were microbubbles, US imaging in destructive mode was used. This mode destroyed some of the remaining MB resulting in fewer bright structures, indicating that the bright structures seen were microbubbles. 


\subsection{Luciferase assay measurement}

Mice were euthanized by lethal $\mathrm{CO}_{2} 5$ days after sonoporation. Achilles tendons were harvested and frozen in liquid nitrogen before lysis could occur. Then the tendons were crushed in liquid nitrogen using a mortar and pestle. The powder was added into an ice cold luciferase Cell Culture Lysis buffer (Promega, Madison, WI) and the mixture was kept for $3 \mathrm{~h}$ on ice before luminescence measurement. The Relative Light Units (RLU) was measured using a Lumat LB9507 luminometer (Berthold, Wildbarch, Germany) after adding $100 \mu$ of Luciferase assay system substrate (Promega) to $20 \mu \mathrm{l}$ of tendon lysates. Luciferase activity was expressed as RLU per mg of proteins. The tendon protein content was quantified by bicinchoninic acid assay [40].

\subsection{Histological analysis}

The sonoporation cytotoxicity was evaluated by histological examination of the tendon tissue. The Achilles tendons were insonified after injection of $10 \mu \mathrm{g}$ pLuc solution with or without BR14 microbubbles at $200 \mathrm{kPa}$ and $400 \mathrm{kPa}$ for $10 \mathrm{~min}$. The tendons were harvested at day 7 after insonation and were fixed in $4 \%$ p-formaldehyde solution, paraffin embedded, sliced in serial sections $(2 \mu \mathrm{m})$, mounted on glass slides and counterstained using Hematoxylin-Eosin-Safranin (HES). The tendon sections were analyzed by a pathologist and cytotoxicity was evaluated according to the presence and extent of inflammatory infiltrates and necrosis.

\subsection{In vivo bioluminescence imaging and quantification}

Bioluminescence imaging was captured using a cooled CCD camera mounted in a dark box chamber with a camera controller, a camera cooling system and a computer for data acquisition and analysis (Hamamatsu Photonics K.K., Hamamatsu City, Japan). Signal intensity was recorded $2 \mathrm{~min}$ after $100 \mu \mathrm{g}$ luciferin injection in the tendon area and quantified as the mean of photons per second of time exposure within the region of interest (ROI) over the tendon.

\subsection{DNA extraction, plasmid rescue assay and quantification}

Mice were euthanatized 25 and 108 days after insonation and their Achilles tendons were harvested and lysed using a modified Hirt method which preferentially extracts circular DNA [41]. Tendons were lysed in $300 \mu \mathrm{l} 10 \%$ Chelex solution and $30 \mu \mathrm{l}$ of proteinase $\mathrm{K}$ (working concentration: $100 \mu \mathrm{g} / \mathrm{ml}$ ) at $56{ }^{\circ} \mathrm{C}$ for $2 \mathrm{~h}$. DNA was ethanol-precipitated and redissolved in $300 \mu \mathrm{l}$ of TE buffer. Two phenol chloroform extraction steps followed by ethanol precipitation of the DNA was then carried out. The final pellet was dissolved in $60 \mu \mathrm{l}$ TE buffer containing RNAse $(50 \mu \mathrm{g} / \mathrm{ml})$. The DNA quantity was evaluated using a NanoDrop spectrophotometer (Thermo Fischer, Illkirch, France). The high efficiency E. coli Dh5 $\alpha$ competent bacteria (New England Biolabs, Ozyme, St Quentin en Yveline, France) were transformed with $150 \mathrm{ng}$ of extracted DNA. Plasmid DNA was extracted from transformed colonies and put on agarose gel.

\subsection{RNA extraction of Fmod KO mice and $q P C R$}

Seven days post-treatment, Fmod KO mice tendons and control wild type tendons were harvested and frozen immediately in liquid nitrogen and then stored at $-80{ }^{\circ} \mathrm{C}$. Tendons were thawed only once to preserve the RNA. The tendons were crushed in liquid nitrogen using a mortar and a pestle (average mass $10 \mathrm{mg}$ ). Sample RNA extractions were performed using an RNA II NucleoSpin ${ }^{\circledR}$ mini kit (Machery-Nagel EURL, Hoerdt, France) which includes a DNAse treatment. In order to insure there was enough RNA material, every extraction was done with a pool of 2 tendons $(10 \mathrm{ng} / \mu \mathrm{l}$, for a total mass of $600 \mathrm{ng}$ of RNA). The RNA quantification was performed using a NanoDrop spectrophotometer (Thermo Fischer). The RNA integrity was checked using a BioAnalyzer 2100 (Agilent Technologies UK Limited, Wokingham, Berkshire, UK) was acceptable when RNA integrity number was more than 7 [42]. For all experiments, $100 \mathrm{ng}$ of RNA was used for reverse transcription using 200 units of M-MLV Reverse Transcriptase (New England Biolabs) for $1 \mathrm{~h}$ at $37^{\circ} \mathrm{C}$. The Fmod gene expression was evaluated from $100 \mathrm{ng}$ of cDNA by qPCR using a Light Cycler II 480 (Roche Applied Science, Meylan, France) with $0.1 \mu \mathrm{M}$ of Fmod specific primers (forward: 5'-CCCTCCAGAT CCCCGCGACT-3', reverse: 5'-CGGGAGGGAACGAAGGGCAG-3') and compared to GAPDH housekeeping gene expression (detected transcript: NM_008084, QIAGEN). The Fmod primers were specific to the human fibromodulin cDNA inserted into the pFmod plasmid.

\subsection{Electron microscopy}

Ultrastructural analysis was done on Achilles tendons of 3 groups of Fmod $\mathrm{KO}$ mice and compared to untreated wild type mice, untreated Fmod $K O$ mice. The 1 st group was injected with $10 \mu \mathrm{g}$ of pFmod alone, the 2nd group was injected with pFmod and insonified $(1 \mathrm{MHz}$, $200 \mathrm{kPa}, 40 \%$ duty cycle, $10 \mathrm{~min}$ ) and the $3 \mathrm{rd}$ group was injected with pFmod and MB and insonified (1 MHz, $200 \mathrm{kPa}, 40 \%$ duty cycle, $10 \mathrm{~min})$.

Achilles tendons from the controls and treated mice were harvested 10 days after treatment and fixed in $4 \% p$-formaldehyde and $1 \%$ glutaraldehyde in $0.1 \mathrm{M}$ phosphate buffer ( $\mathrm{pH} 7.2$ ) for $48 \mathrm{~h}$. The specimens were post-fixed in $2 \%$ osmium tetroxide for $1 \mathrm{~h}$ (Electron Microscopy Science, Hatfield, PA) and dehydrated in a graded series of ethanol solutions (from $50 \%$ to $100 \%$ ) and propylene oxide. The samples were then embedded in epoxy resin LX 112 (SIGMA-Aldrich Co.), incubated for $24 \mathrm{~h}$ at $37^{\circ} \mathrm{C}$ and the resin was polymerized for $48 \mathrm{~h}$ at $60^{\circ} \mathrm{C}$. Sixty micrometer sections were cut transversely using an Ultracut UCT ultramicrotome (Leica) with respect to the main axis of collagen fibers, stained with $5 \%$ uranyl acetate, and placed on electron microscopy one-slot grids coated with Formvar film. The sections were then observed at $100 \mathrm{kV}$ with a Jeol 1011 transmission electron microscope (JEOL Ltd, Tokyo, Japan) connected to a Gatan digital camera driven by Digital Micrograph software (Gatan, Pleasanton, CA). Fibril size analyses was done with NIS-Elements D software (Nikon, Tokyo, Japan). Surface, perimeter and diameter of at least 200 fibers were measured for each group. The level of fiber circularity was determined by the Uzbekov index ( $U$ index) calculated by the following equation:

$$
U=\frac{P}{2 \sqrt{\pi \times S}},
$$

where $P$ is perimeter and $S$ is the surface area of fibrils on perpendicular sections. A U index of 1 corresponds to a perfect circle.

\subsection{Statistical analysis}

Data was expressed as mean \pm SEM or SD. Statistical differences between groups was analyzed by the unilateral Mann-Whitney $U$-test test using XLStat 2007 software (Addinsoft, Paris, France), and the significance was defined as a $p$-value $\leq 0.05$.

\section{Results and discussion}

\subsection{Optimal acoustic parameters for efficient gene transfer in Achilles tendons}

We have tested several conditions to determine the optimal acoustic parameters at $1 \mathrm{MHz}$ US that allow an efficient gene transfer in wild type mice Achilles tendons [39]. The transfection efficiency was evaluated with a plasmid DNA encoding the luciferase reporter 
gene (pLuc), and luciferase activity was used as a read out. Fig. 1 summarizes the luciferase activities obtained in tendons that have been sonoporated after 5 days at $1 \mathrm{MHz}, 200 \mathrm{kPa}$ or $400 \mathrm{kPa}, 40 \%$ duty cycle during 2, 5 or 10 min exposure time in the presence or absence of BR14 bubbles. After 2 min of insonation at $200 \mathrm{kPa}$ peak negative pressure in the presence of $5 \times 10^{5} \mathrm{BR} 14 \mathrm{MB}$ did not enhance the luciferase expression compared to the control condition consisting of injection of pLuc alone. Increasing the exposure time to $5 \mathrm{~min}$ did not have any significant effect. However, 10 min of exposure using the same acoustic parameters resulted in the highest luciferase activity $\left(6.27 \times 10^{7} \mathrm{RLU} / \mathrm{mg}\right.$ proteins versus $4.72 \times 10^{5} \mathrm{RLU} / \mathrm{mg}$ proteins) which is 132 -fold more compared to that obtained with pLuc injected alone. This increase was dependent on the presence of microbubbles as no significant increase in gene expression was found at the same acoustic condition without BR14 MB. Moreover, there was significant increase in gene transfer efficiency after insonation without MB independent of the exposure duration.

In contrast, the resulting trend was quite different when the tendon was insonified at $400 \mathrm{kPa}$. When the tendon was US-exposed for $2 \mathrm{~min}$ in the presence of $\mathrm{MB}$, there was an increase of the gene expression as found for $200 \mathrm{kPa}$. The efficiency of gene transfer was 80 -fold more than that of pLuc alone. However, increasing the exposure time to 5 and $10 \mathrm{~min}$ resulted in reduced luciferase activity.

To check if sonoporation could alter the pDNA structure, agarose gel elecrophoresis of the insonified samples was performed (Supplementary Fig. S1). Fig. 2 shows the electrophoresis results of two insonified samples, a control sample and the DNA ladder. Independent of the acoustic pressure, the pDNA samples resolved in two bands; corresponding to the supercoiled and circular DNA similar to those seen in the non-treated pDNA (the control sample). No smears were observed indicating no degradation. Consequently, the reduced gene expression at $400 \mathrm{kPa}$ was not due to pDNA degradation. Histological analysis was performed in order to assess if this decreased gene expression was due to cytotoxicity of this applied US condition. As depicted in Fig. 2, the examination of HES staining sections clearly indicates that tendons exposed to US at $400 \mathrm{kPa}$ showed specific changes compared to the untreated tendons. A large area of inflammation, characterized

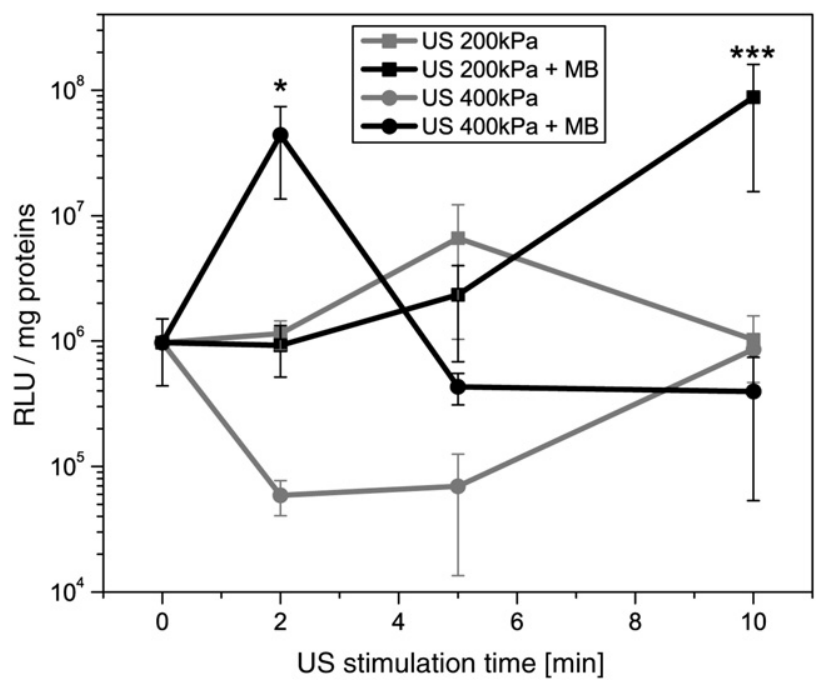

Fig. 1. Sonoporation in mice Achilles tendon: optimal parameters determination. Ten micrograms of plasmid encoding luciferase gene (pLuc) in the absence (gray curve) or the presence (black curve) of $5 \times 10^{5}$ BR14 MB in $10 \mu \mathrm{l}$ final volume was injected at mid region of Achilles tendons. After injection, tendons were exposed either to $1 \mathrm{MHz}$ US at $200 \mathrm{kPa}$ (square) or $400 \mathrm{kPa}$ (circle) during 2, 5 or $10 \mathrm{~min}$. Tendons were harvested 5 days post-sonoporation and the luciferase activity was measured and expressed as Relative Light Units per $\mathrm{mg}$ of proteins (RLU/mg proteins). Values are means \pm SEM of at least 4 tendons. ${ }^{*}: p$-value $<0.05 ;{ }^{* * *}: p$-value $<0.001$ by the presence of a high number of lymphocytes and plasmocytes surrounding the tendon particularly in the musculotendinous junction and the sheath was seen. However, the tendon itself seemed to be unaltered. The observed inflammation was enhanced when the insonation was performed in the presence of MB. Acute inflammatory lesions rich in polynuclear neutrophils and muscle necrosis at the musculotendinous junction were observed (Fig. 2C and E). Although not as extensive, inflammation was evident in tendons exposed at $400 \mathrm{kPa}$ for $2 \mathrm{~min}$ (not shown). This condition gave a 10 -fold more gene expression in tendons compared to the control (pDNA alone) as seen in Fig. 1. By contrast, no significant histopathological changes were seen after insonation at $200 \mathrm{kPa}$ independent of the MB presence, the architecture of tendons was preserved and no inflammatory infiltrates were detected.

\subsection{Kinetic of transgene expression}

After injection, a hyper echogenic structure limited to the tendon area, which induced shadowing artifacts was observed, indicating that microbubbles were concentrated at the site of injection, shown in Fig. 3. After $1 \mathrm{MHz}$ insonation, the same bright structures were still seen, although its size and localization was changed (data not shown).

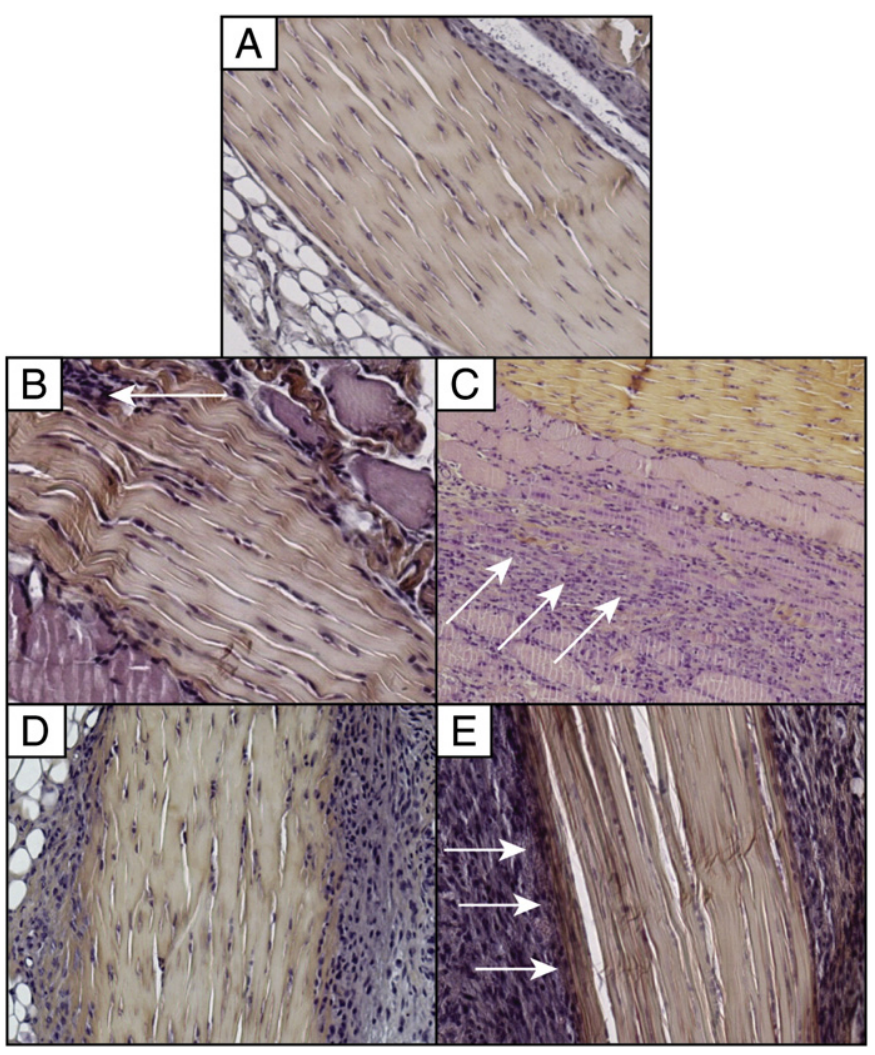

Fig. 2. Histopathological analyses of sonoporated mice Achilles tendons. Histopathological analyses of sonoporated mice Achilles tendons 7 days after treatment. The Achilles tendons were harvested, fixed, paraffin embedded, sliced in serial sections $(2 \mu \mathrm{m})$, mounted on glass slides and HES counterstained. (A) Control tendon: tendon sheath, adipocytic tissue and tendon are healthy without inflammatory infiltrates. (B) Tendon injected with pLuc followed by $200 \mathrm{kPa}$ insonation for $10 \mathrm{~min}$ : no significant modification of the histological structures was seen. The musculotendinous junction can be seen (arrows); (C) tendon injected with pLuc followed by $400 \mathrm{kPa}$ insonation for $10 \mathrm{~min}$ : moderate to intense inflammatory infiltrates were localized in the muscle and focal muscular necrosis (indicated by arrows); (D) tendon injected with pLuc and microbubbles followed by $200 \mathrm{kPa}$ insonation for 10 min: moderate inflammatory infiltrate in the tendon sheath without modification of the tendon structure; (E) tendon injected with pLuc and microbubbles followed by $400 \mathrm{kPa}$ insonation for $10 \mathrm{~min}$ : acute inflammation was seen in the tendon sheath infiltrating the tendon (arrows). 


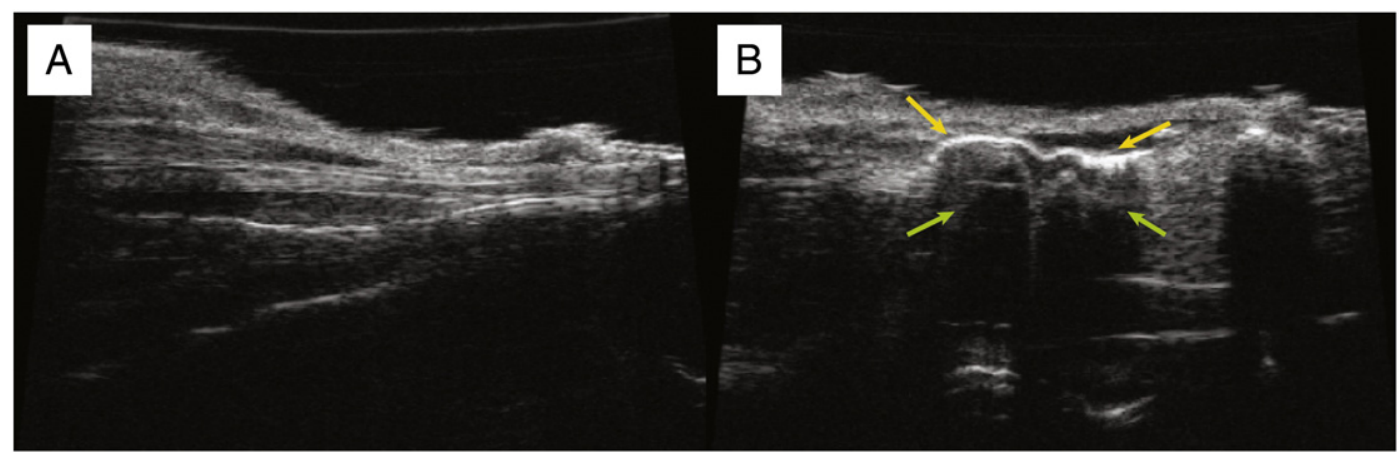

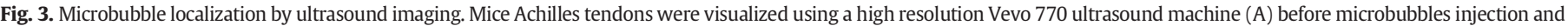

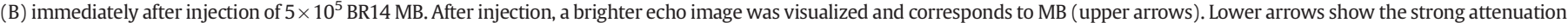
induced by the high concentration of MB (shadowing artifact). Microbubbles are located in the Achilles tendon area only.

Fig. 4 shows in vivo bioluminescence imaging performed on mice injected with $10 \mu \mathrm{g}$ of pLuc alone, or $10 \mu \mathrm{g}$ of pLuc mixed with $5 \times 10^{5} \mathrm{MB}$. In these experiments, the contralateral tendons of treated mice were used as a control. At the indicated times, mice were imaged and the cooled charge-coupled device signal depicted was quantitatively analyzed to give the gene expression profile shown in Fig. 4. From day 1 to day 25 , the relative luciferase activity exhibited in tendons treated with US at $200 \mathrm{kPa}$ and MB during 10 min was 10-fold higher than that obtained with tendons injected with pLuc alone. The difference in the kinetic of gene expression obtained with and without MB can be ascribed to MB oscillations under US application. MB oscillations are known to affect the plasma membrane by inducing shear-stress effects and transient permeabilization. These events increase pDNA internalization and thus the chance to have a longer luciferase expression. At day 25 , it was $1.25 \times 10^{7}$ versus $1.63 \times 10^{5} \mathrm{p} / \mathrm{s} ; p$-value $<0.001$. The
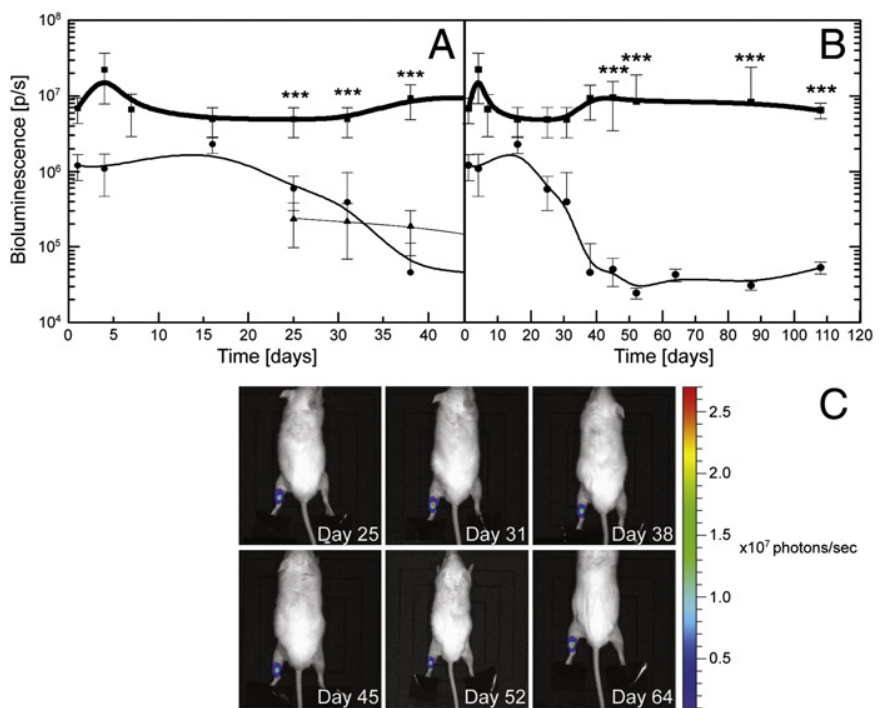

C

Fig. 4. Kinetic of luciferase gene expression. Mice Achilles tendons were injected either with $10 \mu \mathrm{g}$ pLuc only or $10 \mu \mathrm{g}$ pLuc mixed or not with $5 \times 10^{5}$ of BR14 MB followed by US exposure at $1 \mathrm{MHz}, 200 \mathrm{kPa}, 40 \%$ duty cycle during $10 \mathrm{~min}$. Bioluminescence signals (expressed in $\mathrm{p} / \mathrm{s}$ : photons per second) that correspond to the luciferase activity were measured after luciferin injection in the tendon area. (A) and (B): luciferase gene expression profile of mice groups injected with either pLuc only (circle), pLuc mixed with MB and exposed to US (square) or pLuc without MB and exposed to US (triangle). Values are means $\pm S E M$. Data are representative of two experiments with five mice per group. ( ${ }^{* * *}$ : $p$-value $<0.001$ ). (C) Representative CCD images showing luciferase expression in tendons injected with pLuc mixed with MB and US exposed (left tendon) or pLuc without MB and not US exposed (right tendon). Images were recorded at day (D) $25,31,38,45,52$ and 64 post-injection. following days, the level of luciferase activity in tendons injected with pLuc alone decreased gradually to $10^{3} \mathrm{p} / \mathrm{s} 40$ days post-treatment. The luminescence measured in tendons treated with US alone was similar to that of those injected with pLuc alone. This is in agreement with the ex vivo measurement of luciferase activity shown in Fig. 1.

The luciferase expression was 130-fold higher with MB than without MB but only 10-fold increase when measured by bioluminescence imaging. This was due to the sensitivity difference in measuring the bioluminescence emitted between the two techniques used in the experiments. In vivo, the luciferin was injected locally in the tendon area which is poorly vascularized, and thus hypoxic which likely explains the discrepancy between the ex vivo and the in vivo measurements.

Similar long US exposure time has been reported by Sheyn et al. [18] who have transfected mice skeletal muscle by US assisted with OptisonTM bubbles. Their data indicate that luciferase transgene delivery was optimal with the following US parameters: $1 \mathrm{MHz}$, $5 \mathrm{~W} / \mathrm{cm}^{2}, 50 \%$ duty cycle during $10 \mathrm{~min}$.

Fig. 4B shows the tendon luciferase activity after 10 min insonation. The bioluminescence of the tendons insonified with pLuc and MB present was stable for over 100 days as shown by the plateau.

\subsection{Assessment of intact plasmid rescue assay}

The long lasting gene expression obtained could be due to the maintenance of pDNA extra-chromosomally. The pDNA extracted from insonified tendons at optimal acoustic conditions and control tendons was used to transform E. coli. Fig. 5 shows that only DNA extracted from tendons insonified with pLuc and MB was able to transform the E. coli. Even though the number of colonies obtained was low, this result indicates that an intact form of pLuc could be recovered from these tendons; this was not the case for those injected with pLuc alone.

\subsection{Restoration of fibromodulin gene expression in Fmod KO mice}

The last step of our study was to test if the optimal insonation conditions could restore a knocked out gene in Achilles tendons. This investigation was carried out in fibromodulin knock-out mice (Fmod KO) [43]. This mice model had been generated to better understand the role of fibromodulin (Fmod), a small leucin-rich repeat proteoglycan. Fmod is abundantly expressed in tendons and it is known to act on collagen fibril assembly and maturation. Compared to the wild type mice, the average diameter of collagen fibrils of Fmod $\mathrm{KO}$ mice is lower due the higher number of small diameter fibrils [44].

The Achilles tendons of these mice were insonified as described previously with pDNA encoding fibromodulin gene (pFmod). We quantified the gene transfection efficiency by measuring the Fmod gene expression by quantitative RT-PCR. The tendons were harvested and their total RNA was extracted 7 days after insonation. In the insonified mice the 


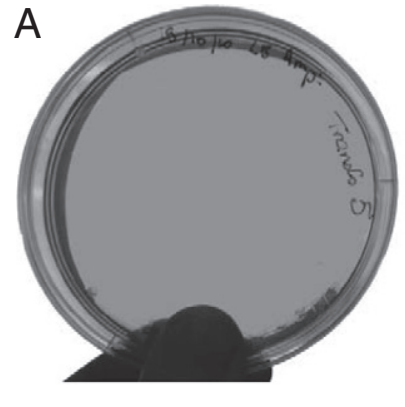

C
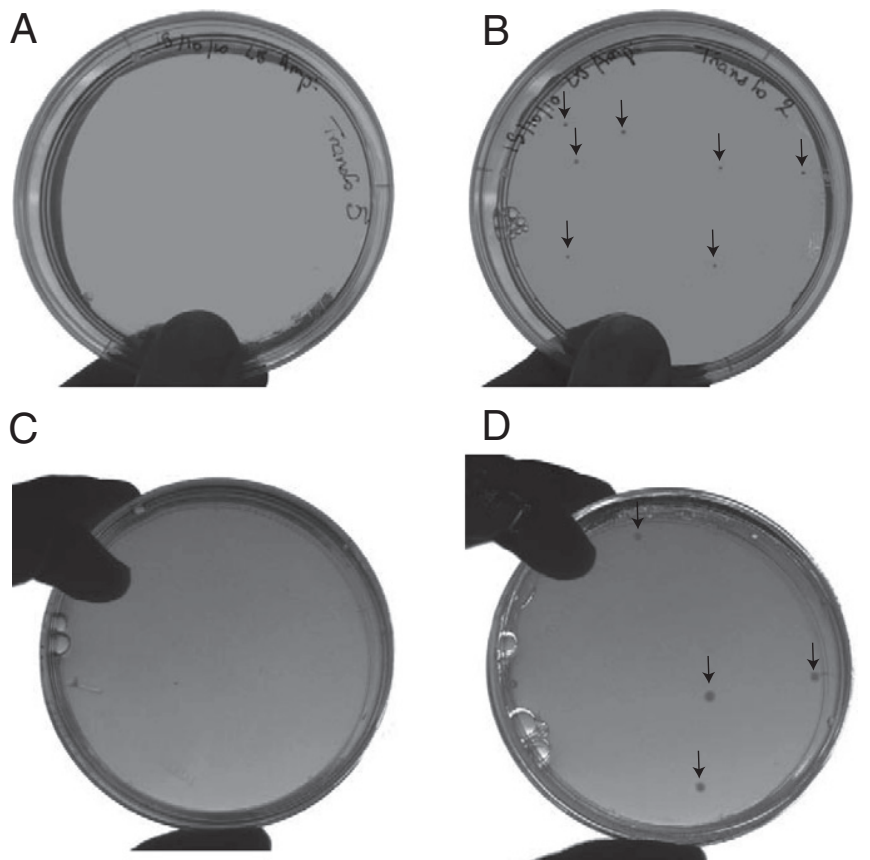

$\mathrm{D}$

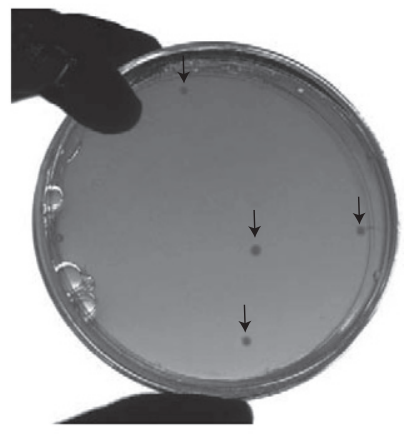

E

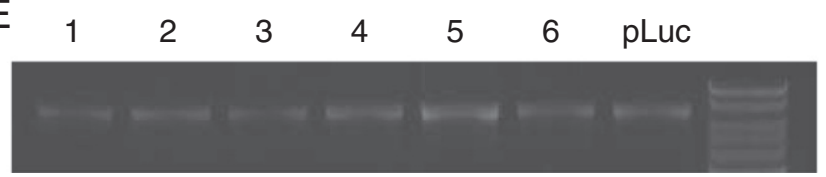

Fig. 5. Plasmid rescue assay. Mice Achilles tendons were treated as described in Fig. 4 They were harvested at day 25 ( $A$ and B) or at day 108 (C and D) post-injection. Then, a modified Hirth method was performed to extract circular DNA. The resulting DNA was used to transform E. coli bacteria. Shown are representative images obtained from the different samples. (A and C): bacterial transformation using DNA from tendons injected with pLuc alone; ( $B$ and $\mathrm{D}$ ): bacterial transformation using DNA from tendons sonoporated with pLuc (pLuc + US + BR14). (E): agarose gel electrophoresis of minipreps (lanes 1 to 6) made from six transformed bacterial colonies (arrows in B and D), lane 7: pLuc; lane 8: DNA ladder.

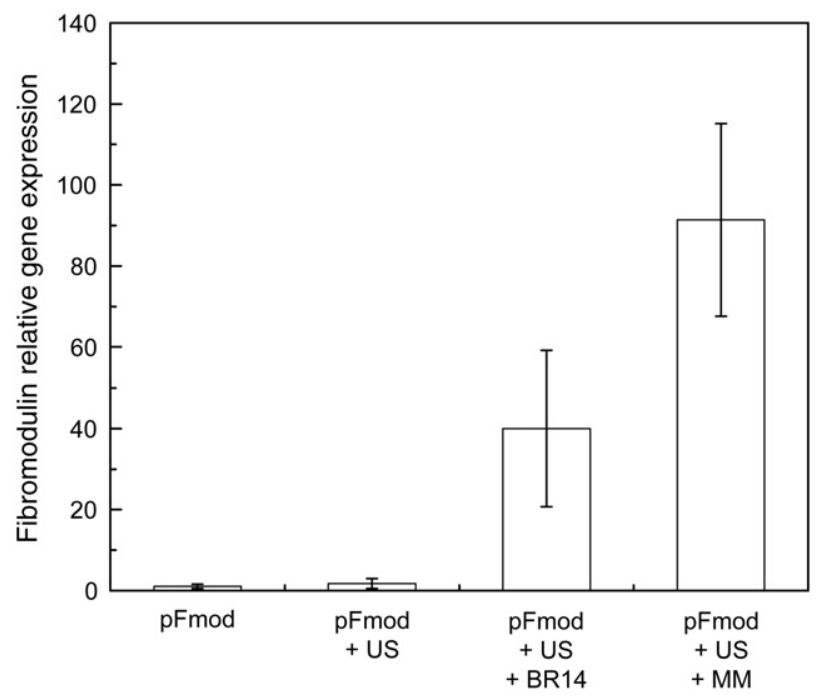

Fig. 6. Fmod gene expression relative to GAPDH. Achilles tendons of Fmod KO mice were injected with $10 \mu \mathrm{g}$ of plasmid encoding for fibromodulin gene (pFmod) with US stimulation in the absence (pFmod +US) or in the presence of BR14 and Micromarker MB $(\mathrm{pFmod}+\mathrm{US}+\mathrm{BR} 14$ and pFmod + US + MM) at optimal acoustic parameters. Achilles tendons were harvested 7 days post-injection and the total RNA was extracted. The relative Fmod gene expression was determined by quantitative qPCR as compared to that of GAPDH housekeeping gene and the untreated Fmod KO mouse tendons are considered as calibrator tissue. Values shown are means \pm SD of 4 tendons. relative expression of Fmod mRNA was 40-fold more than that of GAPDH housekeeping gene while no significant increase was obtained in contralateral tendons injected with pFmod and exposed to US as shown in Fig. 6.

Fig. 7 shows collagen fibrils of Achilles tendons from wild type, nontreated Fmod $\mathrm{KO}$ mice and Fmod $\mathrm{KO}$ mice that were treated either with pFmod alone, pFmod and US or pFmod + MB and US. Collagen fibrils of Fmod $\mathrm{KO}$ mice exhibited an abnormal disorganized structure and irregular shape (Fig. 7B) compared to those of wild type, which were smooth and regular (Fig. 7A) as previously shown [36]. Injection of pFmod followed by US application seemed to induce a slight improvement of collagen fibrils ultrastructure, which was more regular but the occurrence of thinner fibrils was still high (Fig. 7D). By contrast, injection of pFmod alone did not significantly improved collagen fibrils albeit some slight difference when compared to the Fmod $\mathrm{KO}$ mice. It is worth to note that no effect was observed when tendons were treated with US alone or US and MB without pFmod injection (data not shown). Among the different conditions used, it can be seen that sonoporation with an Fmod gene resulted in a dramatic improvement of collagen ultrastructure (Fig. 7E).

These differences were validated by the analysis of collagen fibril diameter distributions of each sample and their Uzbekov index (U index) (Figs. 8 and 9). Fig. 8 shows that the distribution of collagen fibers diameter of Fmod KO mice treated with pFmod and US alone (Fig. 8D) or with microbubbles (Fig. 8E) was similar to that of wild type mice (Fig. 8A). Moreover, the Uzbekov index calculated for each class of collagen fiber diameter indicated that the level of fiber circularity from sonoporated Fmod $\mathrm{KO}$ mice was close to 1 as observed
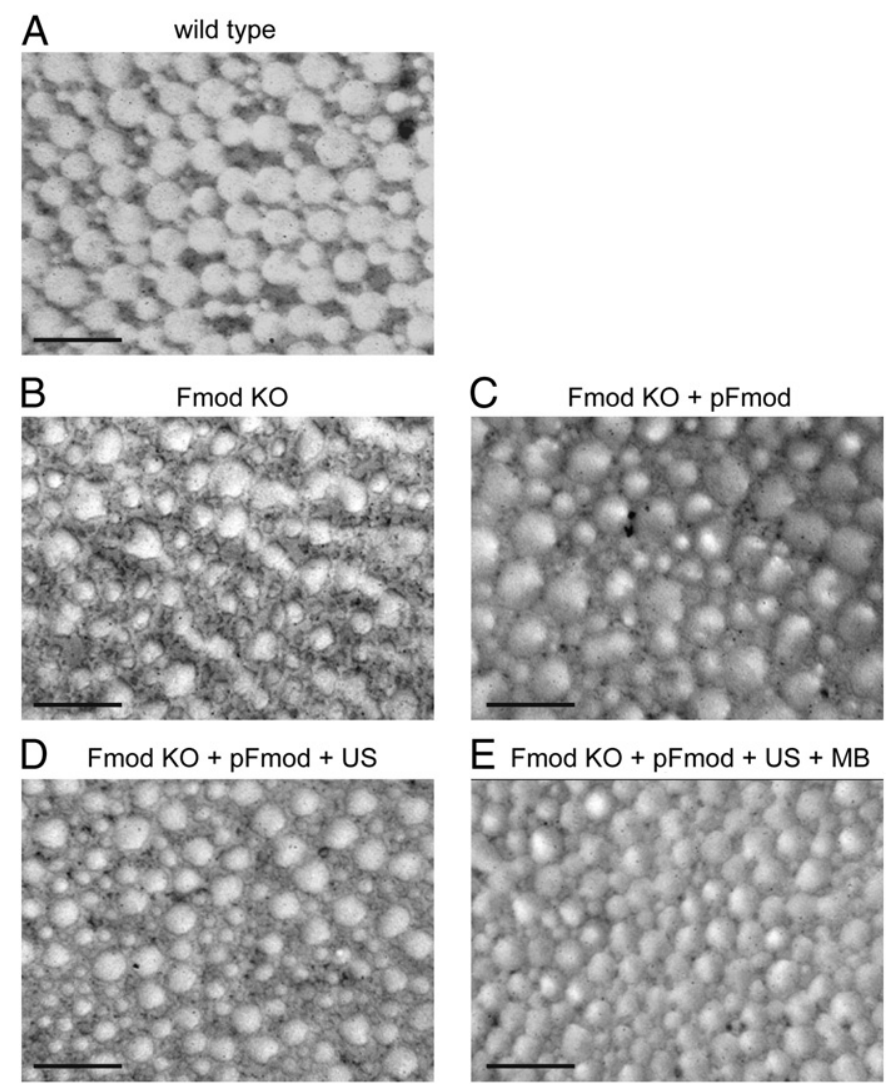

Fig. 7. Effect of Fmod gene expression on collagen fibrils ultrastructure. Transmission electron micrograph images showing the effect of pFmod, microbubbles and ultrasound on Achilles tendons of Fmod KO mice 10 days after treatment. (A) wild type mice tendon; (B) Fmod KO mice tendon; (C) Fmod KO mice tendon injected with pFmod (D) Fmod KO mice tendon injected with pFmod and insonified at optimal acoustic parameters; (E) Fmod KO mice tendon injected with pFmod and Micromarker microbubbles then insonified at optimal acoustic parameters. Magnification 50000 $\times$ Scale bar represents $500 \mathrm{~nm}$. 


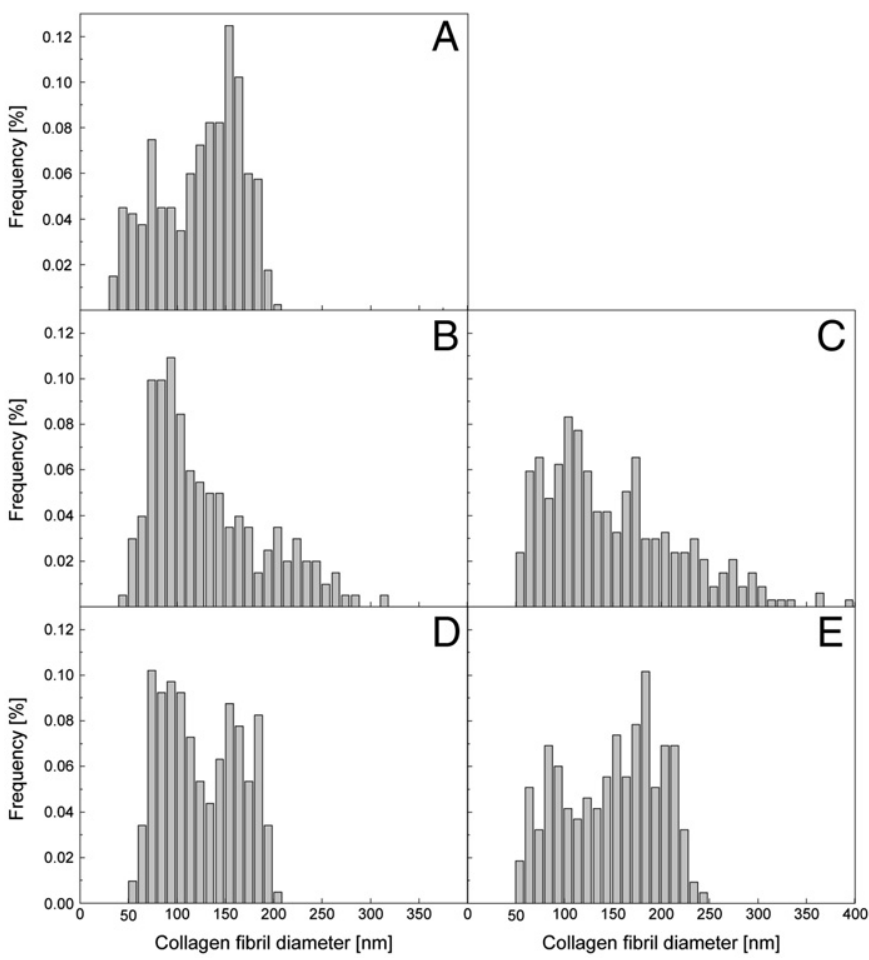

Fig. 8. Ultrastructural analysis of collagen fibrils. Histograms of collagen fibril diameter distributions: (A) wild type, (B) Fmod KO mice, (C) Fmod KO mice injected with pFmod alone, (D) Fmod KO mice injected with pFmod followed by ultrasound stimulation, (E) Fmod KO mice injected with pFmod and Micromarker microbubbles followed by ultrasound stimulation.

for wild type mice (Fig. 9). Note that the value of Uzbekov index for Fmod $\mathrm{KO}$ mice was increased as function of collagen fiber diameter.

\section{Conclusion}

This study shows the potential of US assisted delivery to efficiently transfer a gene into the Achilles tendon without noticeable toxicity. Our results firmly indicate that MB were essential in this US-facilitated gene delivery. We show the influence of microbubbles, acoustic pressure and ultrasound exposure time on gene transfer efficiency. Plasmid with $\mathrm{MB}$ alone did not produce any enhancement suggesting the crucial effect of US trigger.

We found that 10 min of US exposure time was required for optimal gene transfer. The luciferase activity peaked around 1 week and became undetectable 19 days after insonation. Any effect of US alone on gene transfer in Achilles tendons was seen. This absence of US effect is not commonly observed.

Our results indicate that our insonation conditions were successful in delivering sufficient pDNA which was maintained in an intact form for a long period of time leading to sustained gene expression. This stable gene expression could also be related to the fact that the low proliferative index of tenocytes may retain introduced pDNA for a long time, similar to skeletal muscle cells. Compared to data reported in the literature, the gene transfer efficiency of this method ( 100 days) is comparable to that obtained in tendons transduced with adenoviral vectors ( 75 days). Interestingly, the efficiency is clearly superior to that obtained with electroporation which leads to a modest improvement of the gene transfer (by $50 \%$ ) when optimal electric conditions of $200 \mathrm{~V}$ for $10 \mathrm{~ms}$ or $1200 \mathrm{~V}$ for $100 \mu$ s are applied as reported Jayankura et al. [23]. Moreover, the transgene expression lasts only for 42 days which is quite close to that observed with pDNA injection alone as we have obtained in Fig. 4 . The dense structure of tendons could be not suitable with the classical electrotransfer method.

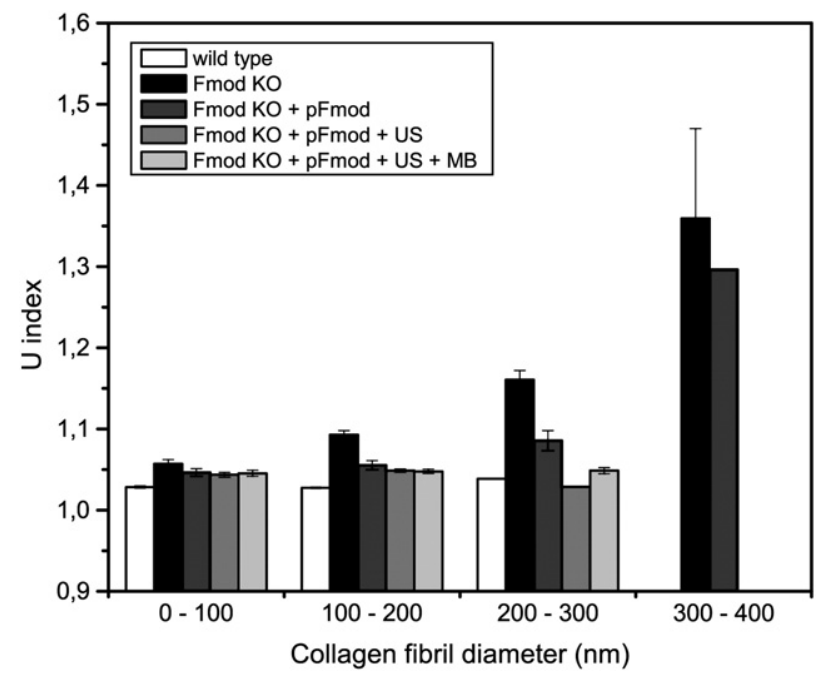

Fig. 9. Uzbekov index of collagen fibers. The level of fiber circularity of collagen fibrils from samples used in Figs. 7 and 8 was evaluated by calculating the Uzbekov index. The index of each class of collagen fibril diameter was calculated. An index of 1 corresponds to a perfect circle.

In the acoustic conditions used in our study, oscillations of MB are likely inducing a mechanical stress on the plasma membrane. In our case, a long lasting gene expression occurred only after 10 min US exposure. This gene delivery method looks promising to restore missing gene or malfunctioning gene that is observed in severe tendon diseases. The potential of this method is validated by the improvement of collagen fibrils phenotype observed 10 days post-treatment. The therapeutic benefit of this gene expression needs to be validated by the determination of biomechanical parameters. Experiments are in progress to define the kinetic of Fmod gene expression and to determine a robust protocol of gene restoration.

This delivery system could be applied to transfer therapeutic genes to accelerate tendon healing or to restore degenerative tendons.

Supplementary materials related to this article can be found online at doi:10.1016/j.jconrel.2011.08.020.

\section{Acknowledgments}

This work was supported by the Region Centre and the CNRS (Centre National de la Recherche Scientifique). A.D. received a Ph.D. fellowship from the Region Centre and the AFSED (Association Française des Syndromes Ehlers-Danlos).

The authors would like to acknowledge Bracco Research (Geneva, Switzerland) for BR14 bubbles, Loïc Lebegue for his skilful technical help and Dr Frédéric Maitre (Centre Hospitalier Régional d'Orléans, France) for histological analyses. They are grateful to Dr Äke Oldberg (Lund University, Sweden) for providing Fmod KO mice and to $\mathrm{Dr}$ Peter Roughley for providing the pCEP4-FBM plasmid.

\section{References}

[1] S. Bao, B.D. Thrall, D.L. Miller, Transfection of a reporter plasmid into cultured cells by sonoporation in vitro, Ultrasound Med. Biol. 23 (1997) 953-959.

[2] D.L. Miller, S. Bao, J.E. Morris, Sonoporation of cultured cells in the rotating tube exposure system, Ultrasound Med. Biol. 25 (1999) 143-149.

[3] Y. Taniyama, K. Tachibana, K. Hiraoka, T. Namba, K. Yamasaki, N. Hashiya, M. Aoki, T. Ogihara, K. Yasufumi, R. Morishita, Local delivery of plasmid DNA into rat carotid artery using ultrasound, Circulation 105 (2002) 1233-1239.

[4] M. Postema, O.H. Gilja, Ultrasound-directed drug delivery, Curr. Pharm. Biotechnol. 8 (2007) 355-361.

[5] A. van Wamel, A. Bouakaz, M. Versluis, N. de Jong, Micromanipulation of endothelial cells: ultrasound-microbubble-cell interaction, Ultrasound Med. Biol. 30 (2004) 1255-1258.

[6] S. Kotopoulis, M. Postema, Microfoam formation in a capillary, Ultrasonics 50 (2010) 260-268. 
[7] K. Kaddur, L. Lebegue, F. Tranquart, P. Midoux, C. Pichon, A. Bouakaz, Transient transmembrane release of green fluorescent proteins with sonoporation, IEEE Trans. Ultrason. Ferroelectr. Freq. Control. 57 (2010) 1558-1567.

[8] B.D. Meijering, L.J. Juffermans, A. van Wamel, R.H. Henning, I.S. Zuhorn, M Emmer, A.M. Versteilen, W.J. Paulus, W.H. van Gilst, K. Kooiman, N. de Jong, R.J. Musters, L.E. Deelman, O. Kamp, Ultrasound and microbubble-targeted delivery of macromolecules is regulated by induction of endocytosis and pore formation, Circ. Res. 104 (2009) 679-687.

[9] X. Wang, H.D. Liang, B. Dong, Q.L. Lu, M.J. Blomley, Gene transfer with microbubble ultrasound and plasmid DNA into skeletal muscle of mice: comparison between commercially available microbubble contrast agents, Radiology 237 (2005) 224-229.

[10] S. Mehier-Humbert, T. Bettinger, F. Yan, R.H. Guy, Plasma membrane poration induced by ultrasound exposure: implication for drug delivery, J. Control. Release 104 (2005) 213-222.

[11] C. Pichon, K. Kaddur, P. Midoux, F. Tranquart, A. Bouakaz, Recent advances in gene delivery with ultrasound and microbubbles, J. Exp. Nanosci. 3 (2008) 17-40.

[12] M. Duvshani-Eshet, M. Machluf, Efficient transfection of tumors facilitated by long-term therapeutic ultrasound in combination with contrast agent: from in vitro to in vivo setting, Cancer Gene Ther. 14 (2007) 306-315.

[13] P. Hauff, S. Seemann, R. Reszka, M. Schultze-Mosgau, M. Reinhardt, T. Buzasi, T. Plath, S. Rosewicz, M. Schirner, Evaluation of gas-filled microparticles and sonoporation as gene delivery system: feasibility study in rodent tumor models, Radiology 236 (2005) 572-578.

[14] S. Hayashi, M. Mizuno, J. Yoshida, A. Nakao, Effect of sonoporation on cationic liposome-mediated IFNbeta gene therapy for metastatic hepatic tumors of murine colon cancer, Cancer Gene Ther. 16 (2009) 638-643.

[15] H. Koike, N. Tomita, H. Azuma, Y. Taniyama, K. Yamasaki, Y. Kunugiza, K. Tachibana, T. Ogihara, R. Morishita, An efficient gene transfer method mediated by ultrasound and microbubbles into the kidney, J. Gene Med. 7 (2005) 108-116.

[16] Y. Manome, M. Nakamura, T. Ohno, H. Furuhata, Ultrasound facilitates transduction of naked plasmid DNA into colon carcinoma cells in vitro and in vivo, Hum. Gene Ther. 11 (2000) 1521-1528.

[17] Y. Sakakima, S. Hayashi, Y. Yagi, A. Hayakawa, K. Tachibana, A. Nakao, Gene therapy for hepatocellular carcinoma using sonoporation enhanced by contrast agents, Cancer Gene Ther. 12 (2005) 884-889.

[18] D. Sheyn, N. Kimelman-Bleich, G. Pelled, Y.Zilberman, D. Gazit, Z. Gazit, Ultrasoundbased nonviral gene delivery induces bone formation in vivo, Gene Ther. 15 (2008) 257-266.

[19] W. Wei, Z.Z. Bian, Y.J. Wu, L.F. Yan, Y.L. Miao, A novel approach to quantitative ultrasonic naked gene delivery and its non-invasive assessment, Ultrasonics 43 (2004) 69-77.

[20] K.A. Hildebrand, C.B. Frank, D.A. Hart, Gene intervention in ligament and tendon: current status, challenges, future directions, Gene Ther. 11 (2004) 368-378.

[21] Y. Hou, Z. Mao, X. Wei, L. Lin, L. Chen, H. Wang, X. Fu, J. Zhang, C. Yu, Effects of transforming growth factor-beta1 and vascular endothelial growth factor 165 gene transfer on Achilles tendon healing, Matrix Biol. (2009) 324-335.

[22] J. Lou, In vivo gene transfer into tendon by recombinant adenovirus, Clin. Orthop. Relat. Res. (2000) S252-255.

[23] M. Jayankura, C. Boggione, C. Frisen, O. Boyer, P. Fouret, G. Saillant, D. Klatzmann, In situ gene transfer into animal tendons by injection of naked DNA and electrotransfer, J. Gene Med. 5 (2003) 618-624.

[24] W.R. Walsh, P. Stephens, F. Vizesi, W. Bruce, J. Huckle, Y. Yu, Effects of lowintensity pulsed ultrasound on tendon-bone healing in an intra-articular sheep knee model, Arthroscopy 23 (2007) 197-204.
[25] C.S. Enwemeka, O. Rodriguez, S. Mendosa, The biomechanical effects of lowintensity ultrasound on healing tendons, Ultrasound Med. Biol. 16 (1990) 801-807.

[26] L. Qin, H. Lu, P. Fok, W. Cheung, Y. Zheng, K. Lee, K. Leung, Low-intensity pulsed ultrasound accelerates osteogenesis at bone-tendon healing junction, Ultrasound Med. Biol. 32 (2006) 1905-1911.

[27] Y.C. Chen, L.P. Jiang, N.X. Liu, Z.H. Wang, K. Hong, Q.P. Zhang, P85, Optison microbubbles and ultrasound cooperate in mediating plasmid DNA transfection in mouse skeletal muscles in vivo, Ultrason. Sonochem. 18 (2010) 513-519.

[28] Q.L. Lu, H.D. Liang, T. Partridge, M.J. Blomley, Microbubble ultrasound improves the efficiency of gene transduction in skeletal muscle in vivo with reduced tissue damage, Gene Ther. 10 (2003) 396-405

[29] M.A. Dinno, M. Dyson, S.R. Young, A.J. Mortimer, J. Hart, L.A. Crum, The significance of membrane changes in the safe and effective use of therapeutic and diagnostic ultrasound, Phys. Med. Biol. 34 (1989) 1543-1552.

[30] J.L. Karnes, H.W. Burton, Continuous therapeutic ultrasound accelerates repair of contraction-induced skeletal muscle damage in rats, Arch. Phys. Med. Rehabil. 83 (2002) 1-4.

[31] A.J. Mortimer, M. Dyson, The effect of therapeutic ultrasound on calcium uptake in fibroblasts, Ultrasound Med. Biol. 14 (1988) 499-506.

[32] S.R. Young, M. Dyson, The effect of therapeutic ultrasound on angiogenesis, Ultrasound Med. Biol. 16 (1990) 261-269.

[33] A. Ramirez, J.A. Schwane, C. McFarland, B. Starcher, The effect of ultrasound on collagen synthesis and fibroblast proliferation in vitro, Med. Sci. Sports Exerc. 29 (1997) 326-332.

[34] W. Harvey, M. Dyson, J.B. Pond, R. Grahame, The stimulation of protein synthesis in human fibroblasts by therapeutic ultrasound, Rheumatol. Rehabil. 14 (1975) 237.

[35] Y. Kobayashi, D. Sakai, T. Iwashina, S. Iwabuchi, J. Mochida, Low-intensity pulsed ultrasound stimulates cell proliferation, proteoglycan synthesis and expression of growth factor-related genes in human nucleus pulposus cell line, Eur. Cell Mater. 17 (2009) 15-22.

[36] H. Pan, Y. Zhou, O. Izadnegahdar, J. Cui, C.X. Deng, Study of sonoporation dynamics affected by ultrasound duty cycle, Ultrasound Med. Biol. 31 (2005) 849-856.

[37] A. Suwalski, H. Dabboue, A. Delalande, S.F. Bensamoun, F. Canon, P. Midoux, G Saillant, D. Klatzmann, J.P. Salvetat, C. Pichon, Accelerated Achilles tendon healing by PDGF gene delivery with mesoporous silica nanoparticles, Biomaterials 31 (2010) 5237-5245

[38] Y. Geng, D. McQuillan, P.J. Roughley, SLRP interaction can protect collagen fibrils from cleavage by collagenases, Matrix Biol. 25 (2006) 484-491.

[39] A. Delalande, M.F. Bureau, P. Midoux, A. Bouakaz, C. Pichon, Ultrasound-assisted microbubbles gene transfer in tendons for gene therapy, Ultrasonics 50 (2010) 269-272.

[40] P.K. Smith, R.I. Krohn, G.T. Hermanson, A.K. Mallia, F.H. Gartner, M.D. Provenzano, E.K. Fujimoto, N.M. Goeke, B.J. Olson, D.C. Klenk, Measurement of protein using bicinchoninic acid, Anal. Biochem. 150 (1985) 76-85.

[41] B. Hirt, Selective extraction of polyoma DNA from infected mouse cell cultures, J. Mol. Biol. 26 (1967) 365-369.

[42] A. Schroeder, O. Mueller, S. Stocker, R. Salowsky, M. Leiber, M. Gassmann, S. Lightfoot, W. Menzel, M. Granzow, T. Ragg, The RIN: an RNA integrity number for assigning integrity values to RNA measurements, BMC Mol. Biol. 7 (2006).

[43] S. Chakravarti, Functions of lumican and fibromodulin: lessons from knockout mice, Glycoconj. J. 19 (2002) 287-293.

[44] L. Svensson, A. Aszodi, F.P. Reinholt, R. Fassler, D. Heinegard, A. Oldberg, Fibromodulinnull mice have abnormal collagen fibrils, tissue organization, and altered lumican deposition in tendon, J. Biol. Chem. 274 (1999) 9636-9647. 